\title{
CARACTERÍSTICAS SENSORIAIS E FÍSICO-QUÍMICAS DE GELEIAS MISTAS DE MANGA E ACEROLA
}

\author{
MARIA INÊS SUCUPIRA MACIEL* \\ ENAYDE DE ALMEIDA MELO* \\ VERA LÚCIAARROXELAS GALVÃO DE LIMA* \\ WEDJA SANTANA DA SILVA** \\ CHRISTINE MARIA CARNEIRO MARANHÃO *** \\ KELVINAARAÚJO DE SOUZA****
}

\begin{abstract}
Este trabalho teve como objetivo determinar a melhor formulação de geleia mista de manga espada e acerola e verificar o efeito dos tratamentos sobre as características físico-químicas e sensoriais do produto. Foram elaboradas cinco formulações, contendo diferentes proporções de caldo de manga (M) $(75 \%, 60 \%, 50 \%, 40 \%, 25 \%)$, de acerola (A) $(25 \%, 40 \%, 50 \%, 60 \%, 75 \%)$, pectina $(0,1 \% ; 0,125 \%$; $0,2 \% ; 0,3 \% ; 0,6 \%)$, ácido cítrico $(0,1 \% ; 0,125 \% ; 0,25 \%)$ e açúcar $(30 \%)$. As geleias obtidas foram submetidas a teste sensorial pela método de ordenação - preferência e as formulações de maior preferência ao Teste de Análise Descritiva Quantitativa (ADQ) pelo método perfil de características. A acidez total titulável, $\mathrm{pH}$, ácido ascórbico, sólidos solúveis totais (SST), carotenoides totais, polifenóis (antocianinas, flavonóis, e fenólicos totais) e cor foram determinadas e os resultados submetidos à análise de variância (ANOVA) e ao teste de Tukey ao nível de $5 \%$. Todas as geleias foram classificadas como "extra" uma vez que o teor de SST situou-se entre 63,5 a $64^{\circ}$ Brix. As geleias G7 (75\%M:25\%A), G5 (60\%M:40\%A) e G3 (50\%M:50\%A) foram as mais apreciadas pelos julgadores, destacando-se a G5 e a G7 por reunirem, de forma satisfatória, todos os atributos de qualidade. As geleias mistas apresentaram teor elevado de ácido ascórbico e quantidades significantes de carotenoides e polifenóis. As geleias com maior proporção de manga na formulação apresentaram as melhores características sensoriais e forte potencial antioxidante em função do teor de ácido ascórbico, carotenoides totais e polifenóis. Assim, a acerola (com teores significativos desses fitoquímicos bioativos) pode ser vista como agente enriquecedor na produção de geleias mistas com manga.
\end{abstract}

PALAVRAS-CHAVE: GELEIA MISTA; MANGA; ACEROLA; ANÁLISE SENSORIAL; ÁCIDO ASCÓRBICO; FITOQUÍMICOS.

\footnotetext{
* Professoras adjuntas, Departamento de Ciências Domésticas (DCD), Universidade Federal Rural de Pernambuco (UFRPE) Recife, PE (e-mail: m.ines@dcd.ufrpe.br;eamelo@dcd.ufrpe.br; veraarroxelas@homail.com).

** Mestranda em Tecnologia de Alimentos, Fortaleza, CE (e-mail: wedjasilva2@hotmail.com).

*** Graduada em Economia Doméstica, DCD, UFRPE, Recife, PE (e-mail: tina_maranhao@yahoo.com.br).

**** Professora substituta, DCD, UFRPE, recife, PE (e-mail: kelvina@ig.com.br).
} 


\section{INTRODUÇÃO}

O Brasil é o segundo maior produtor mundial de frutas e o Nordeste destaca-se, principalmente, na produção de manga, uva, banana, goiaba, coco e acerola. A região do Vale do São Francisco, no nordeste brasileiro, é considerada como uma das principais áreas produtoras e exportadoras do país. Em 2005, sua produção de manga atingiu 350 mil toneladas, contribuindo para o desempenho nacional, cuja exportação cresceu 12,99\% e atingiu US $\$ 72$ milhões (BASF, 2006). Apesar do Brasil ser um dos maiores exportadores de manga in natura, a utilização da fruta na industrialização de produtos ainda é insignificante.

A manga, fruta de grande aceitação, apresenta percentual energético superior ao da maçã e ao da laranja, além de constituir rica fonte de pró-vitamina A (caroteno), vitamina B2 (riboflavina), vitamina PP (niacina) e vitamina C (JACOMETTI, MENEGHEL e YAMASHITA, 2003). A variedade Espada, uma das mais cultivadas na Região Nordeste, é consumida em larga escala na forma in natura, cujo sabor e aroma são bastante apreciados.

A Região Nordeste vem se destacando, também, na produção de acerola (Malpighia emarginata DC.) com, aproximadamente, 22.500 toneladas de frutos (BASF, 2006). A acerola apresenta grande importância nutricional, sendo conhecida como fonte natural de vitamina $C$ e de compostos antioxidantes, como carotenoides, antocianinas, flavonóis e fenólicos (KUSKOSKI et al., 2005; LIMAet al., 2003; AGOSTINICOSTA, ABREU e ROSSETTI, 2003). Também faz parte da composição da fruta a tiamina, riboflavina, niacina, proteína e minerais, como sódio, cálcio, potássio e, principalmente, ferro (ASSIS et al., 2002). Tratando-se de fruta altamente perecivel faz-se necessário desenvolver produtos, viáveis economicamente, de modo a torná-la acessível para maior número de pessoas. Além da forma in natura, a acerola pode ser consumida como geleia, suco, licor, xarope, sorvete, doce em calda ou pasta. Em função do seu elevado teor de ácido ascórbico, a fruta também pode ser usada na preservação e no enriquecimento de sucos, néctares, refrigerantes e geleias (BARNABÉ e VENTURINI FILHO, 2004; MATSUURAet al., 2004; ARANHA et al., 2004; MATSUURA e ROLIN, 2002; MELO, LIMA e NASCIMENTO, 1999).

A preocupação mundial com a saúde tem promovido mudanças nos hábitos alimentares da população, aumentando seu interesse pelo valor nutritivo e compostos bioativos presentes nos alimentos processados e exigindo das indústrias produtos de boa qualidade. Entretanto, ainda não foi estabelecido padrão de qualidade para o beneficiamento dessas frutas na agroindústria, visando à produção de sucos, néctares, geleias, sorvetes, chutney, compotas e marmeladas.

A transformação da fruta em produto industrial permite a sua preservação por período prolongado, porém espera-se que as propriedades benéficas ao ser humano e as características sensoriais sejam mantidas ao máximo. Nesse sentido, os testes sensoriais que utilizam os órgãos dos sentidos humanos como "instrumento" de medida, podem ser utilizados no controle de qualidade do produto. Trata-se de medida multidimensional integrada que apresenta vantagens como, por exemplo, determinar a aceitação de produtos por parte dos consumidores (CARDELLO e CARDELLO, 1998).

Este trabalho teve como objetivo determinar a melhor formulação para geleias mistas à base de acerola e manga e averiguar os efeitos dos tratamentos sobre suas características físico-químicas e sensoriais, tendo em vista seu potencial nutritivo e antioxidante.

\section{MATERIAL E MÉTODOS}

Foram utilizadas mangas da variedade Espada (Mangifera indica), provenientes de mercado localizado na cidade de Recife (PE), acerolas (Malpighia emarginata DC.) de acessos cultivados no Banco Ativo de Germoplasma da Universidade Federal Rural de Pernambuco (UFRPE), instalado na Estação Experimental de Cana-de-açúcar de Carpina (PE), e pectina tipo 8105, cedida pela Braspectina S.A. Foram desenvolvidas cinco formulações de geleias contendo diferentes proporções de caldo de Manga $(25 \%, 40 \%, 50 \%, 60 \%$, $75 \%)$, caldo de acerola $(75 \%, 60 \%, 50 \%, 40 \%, 25 \%)$, pectina de alto teor de metoxilação $(0,1 \% ; 0,125 \%$; $0,2 \% ; 0,3 \% ; 0,6 \%)$, açúcar (30\%) e ácido cítrico (0,1\%; 0,125\%; 0,25\%) (Tabela 1). O percentual de pectina e ácido cítrico variou de acordo com as proporções das frutas, reduzindo-se os valores de pectina quando a manga predominava. Essas proporções foram determinadas em estudo preliminar. 
TABELA 1 - FORMULAÇÕES DE GELEIAS SIMPLES E MISTAS DE MANGA COM ACEROLA

\begin{tabular}{ccccccc}
\hline FORM ULA ÇÕ ES & \multicolumn{7}{c}{ PROPORÇ ÕES (\%) } \\
\hline G A & Manga & Acerola & Açúcar & Á gua & Pectina & Ácido Cítrico \\
G M & 0 & 100 & 30 & 0 & 0,3 & 0 \\
G 3 & 100 & 0 & 30 & 40 & 0,125 & 0,125 \\
G 4 & 50 & 50 & 30 & 30 & 0,3 & 0 \\
G 5 & 40 & 60 & 30 & 30 & 0,2 & 0,1 \\
G 6 & 60 & 40 & 30 & 40 & 0,1 & 0,1 \\
G 7 & 25 & 75 & 30 & 0 & 0,6 & 0,25 \\
\end{tabular}

As geleias mistas de manga e acerola foram produzidas em bateladas, em recipientes abertos de acordo com JACKIX (1988). As mangas foram lavadas, descascadas, cortadas, cozidas a $95^{\circ} \mathrm{C}$, com $1 / 3$ de água por 12 minutos e filtradas em gaze para a obtenção do caldo clarificado. $O$ caldo clarificado de acerola foi obtido após lavagem das frutas, mediante cocção a $95^{\circ} \mathrm{C}$ por 12 minutos e filtração em gaze. O pH e o teor de sólidos solúveis dos caldos foram determinados de acordo com os métodos analíticos da AOAC (2002). Os caldos das frutas, misturados nas proporções estabelecidas, foram aquecidos até aproximadamente $95^{\circ} \mathrm{C}$. O "mix", preparado com pectina e açúcar, acrescido de água a $60^{\circ} \mathrm{C}$ foi adicionado e a formulação mantida sob aquecimento até atingir o ponto de geleificação, com teor de sólidos solúveis totais de $63^{\circ}$ brix.

A geleia obtida foi imediatamente transferida para potes de vidro de $250 \mathrm{~mL}$, com tampas metálicas, e resfriada em água corrente. Após quinze dias de armazenamento, em temperatura de $22^{\circ} \mathrm{C}$ a $25^{\circ} \mathrm{C}$ e ausência de luminosidade, as geleias foram submetidas à análise sensorial em cabines individuais, climatizadas, do Laboratório de Análise Sensorial de Alimentos do Departamento de Ciências Domésticas da UFRPE. A equipe constituiu-se de 30 julgadores não treinados, que avaliaram, pelo método de ordenação-preferência, o grau de "gostar" relacionado à geleia de cada formulação. Cada julgador recebeu cinco amostras de geleia mista (aproximadamente $15 \mathrm{~g}$ cada), em copos de polietileno de cor branca, com capacidade de $50 \mathrm{~mL}$, codificadas de maneira casualizada com três dígitos para ordená-las de acordo com sua preferência, colocando o número 1 (amostra de maior preferência), 2 (segunda preferida), etc. de acordo com FERREIRA et al. (2000).

As geleias de maior preferência foram submetidas à Análise Descritiva Quantitativa (ADQ), contando com a participação de dez julgadores treinados (STONE e SIDEL, 1993). Esses julgadores foram selecionados entre alunos, funcionários e professores do Departamento de Ciências Domésticas mediante teste de sensibilidade aos quatro gostos básicos (ácido, amargo, azedo e doce), conforme a metodologia descrita por PENNA (1980). Os indivíduos que apresentaram habilidade para reconhecer os quatro gostos básicos foram selecionados para a etapa de desenvolvimento de terminologia descritiva das amostras de geleias.

O levantamento inicial dos termos descritivos para os atributos de aparência, sabor e consistência foi realizado utilizando-se o método de rede (Kelly's repertory grid method) e princípios básicos do método de análise descritiva quantitativa (STONE e SIDEL, 1993). Geleias de manga com acerola foram apresentadas aos julgadores para que descrevessem as similaridades e diferenças existentes entre elas com relação aos atributos descritos. Após as avaliações individuais, os julgadores foram reunidos e sob a supervisão do líder discutiram os termos gerados para a identificação das características sensoriais relevantes da geleia. Alguns termos correlatos foram eliminados e os sinônimos agrupados. Elaborou-se a lista consensual de termos descritivos com a ajuda de materiais de referência como acerolas maduras aquecidas, mangas maduras aquecidas e solução de ácido cítrico para serem utilizados na obtenção de uniformidade entre os julgadores (Quadro 1).

Com os termos escolhidos foi montada a ficha de avaliação (QUADRO 2) com escalas não estruturadas de $9 \mathrm{~cm}$ e intensidade dos atributos variando de 1 (menor intensidade) a 9 (maior intensidade). 


\section{QUADRO 1 - ATRIBUTOS UTILIZADOS NA ANÁLISE SENSORIAL}

\begin{tabular}{|c|c|}
\hline DESCRTORES & REFERÊNCIAS \\
\hline Aparência & Aspecto superficial das geleias \\
\hline Brilho & Quantidade de luz refletida \\
\hline Fraco & Pouco ou nenhum brilho \\
\hline Forte & Muito brilho \\
\hline Cor & $\begin{array}{l}\text { Sensação produzida pela estimulação da retina pelos raios } \\
\text { luminosos de comprimentos de onda variável }\end{array}$ \\
\hline Clara & Cor vermelho-alaranjado \\
\hline Escura & Cor vermelho-amarronzado \\
\hline Exsudação & Migração de líquidos de dentro para fora da geleia \\
\hline Nenhuma & Ausência de líquido \\
\hline Muita & Presença de líquido \\
\hline Sabor & $\begin{array}{l}\text { Resultado da associação dos materiais utilizados para a } \\
\text { elaboração das geleias }\end{array}$ \\
\hline Acerola & $\begin{array}{l}\text { Sabor de acerola madura processada termicamente com adição } \\
\text { de açúcar }\end{array}$ \\
\hline Manga & $\begin{array}{l}\text { Sabor de manga madura processada termicamente com adição } \\
\text { de acúcar }\end{array}$ \\
\hline Ácido & $\begin{array}{l}\text { Sensação causada por ácidos, sentida principalmente na } \\
\text { superfície da língua }\end{array}$ \\
\hline $\begin{array}{l}\text { Consistêncial } \\
\text { firmeza }\end{array}$ & Menor ou maior facilidade de espalhamento do gel \\
\hline $\begin{array}{l}\text { Pouco firme } \\
\text { Muito firme }\end{array}$ & $\begin{array}{l}\text { Gel mole } \\
\text { Gel firme }\end{array}$ \\
\hline
\end{tabular}

Durante o treinamento, em cabines individuais, os julgadores receberam várias amostras de geleias de manga com acerola, servidas em copos de polietileno de cor branca codificados com três dígitos, quadro de descritores e ficha de avaliação. Para remover o sabor residual entre as amostras, utilizou-se água mineral, em temperatura ambiente e biscoito sem sal. Após a degustação de cada amostra os julgadores assinalaram o ponto na escala que melhor refletia o seu julgamento da intensidade do atributo.

Os resultados de cada julgador para cada atributo foram submetidos à ANOVA e selecionados 10 julgadores que apresentaram bom poder discriminativo $\left(\mathrm{pF}_{\text {amostra }}<0,50\right)$, boa repetibilidade $(\mathrm{pF}$ repetição $>0,05)$ e consenso com a equipe.

Para definição do perfil sensorial das geleias de manga com acerola, três amostras foram testadas (nas mesmas condições descritas anteriormente pelos julgadores selecionados) utilizandose a ficha de avaliação descritiva previamente desenvolvida. Adotou-se planejamento experimental de blocos completos casualizados com apresentação monádica das amostras. Os resultados foram avaliados por ANOVA e Teste de Tukey ao nível de 5\% de significância, utilizando-se o programa Statistic for windows 6.1 (FERREIRA et al., 2000).

Com a finalidade de caracterizar as geleias selecionadas e compará-las com as geleias de manga (100\%) e de acerola (100\%) foram efetuadas as seguintes determinações analíticas: $\mathrm{pH}$, utilizando potenciômetro com eletrodo de vidro; sólidos solúveis totais (SST) em refratômetro de bancada, marca ATAGO, expressos em ${ }^{\circ}$ Brix; acidez total titulável (ATT) e ácido ascórbico (AA) de acordo com métodos analíticos da AOAC (2002); antocianinas e flavonóis totais pelo método espectrofotométrico, proposto por LEES e FRANCIS (1972); carotenoides totais, segundo método descrito por RODRIGUEZ-AMAYA (1999); fenólicos totais de acordo com a metodologia proposta por TSIMIDOU, PAPADOPOULOS e BOSKOU (1992), usando Folin-ciocalteau e catequina como padrão. Para a determinação objetiva da cor, usou-se colorímetro da marca Minolta, operado em sistema CIE $\left(L^{*} a^{*} b^{\star}\right)$, sendo $L^{*}$ a luminosidade, $a^{\star}$ a intensidade da cor vermelha e $b^{\star} a$ intensidade da cor amarela (McGUIRE, 1992). 


\section{QUADRO 2 - ESCALA DE AVALIAÇÃO DOS ATRIBUTOS UTILIZADOS NA ANÁLISE SENSORIAL}

\begin{tabular}{|c|c|c|}
\hline Atributos & \multicolumn{2}{|c|}{ Escala } \\
\hline \multirow{2}{*}{ Brilho } & & \\
\hline & Fraco & Forte \\
\hline \multirow[t]{2}{*}{ Cor } & & \\
\hline & Clara & Escura \\
\hline \multirow[t]{2}{*}{ Exsudação } & & \\
\hline & Nenhuma & Muita \\
\hline \multirow[t]{2}{*}{ Sabor/acerola } & & \\
\hline & Fraco & Forte \\
\hline \multirow[t]{2}{*}{ Sabor/manga } & & \\
\hline & Fraco & Forte \\
\hline Sabor/ácido & & 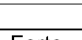 \\
\hline \multirow[t]{2}{*}{ Consistência/firmeza } & Fraco & Forte \\
\hline & Pouco firme & o firme \\
\hline
\end{tabular}

As somas das ordens de cada amostra resultante do teste de preferência foram calculadas e submetidas ao teste de Friedman, utilizando-se os dados da tabela de Newell e MacFarlane. Os dados da ADQ e das características físico-químicas, obtidos a partir do delineamento experimental com blocos completos casualizados e três repetições foram submetidos à análise de variância (ANOVA) e classificação pelo teste de Tukey ao nível de $5 \%$ de probabilidade utilizando-se o programa Statistic for windows 6.1.

\section{RESULTADOS E DISCUSSÃO}

Para a extração do caldo clarificado de manga foi necessária diluição com 30\% de água, devido a sua consistência pastosa em relação à acerola. Frutas firmes requerem adição de água para extração do caldo, porém grandes quantidades devem ser evitadas porque a diluição excessiva da pectina exigirá longas horas de cocção, o que vai reduzir a sua força (JACKIX, 1988). De acordo com o teste de identificação da presença de pectina na fruta (DUTRA, 2000), a acerola apresentou nível baixo e a manga enquadrou-se no nível médio. Concentrações variadas de ácido cítrico foram adicionadas às misturas de modo a ajustar o $\mathrm{pH}$ das formulações.

As geleias mistas G3, G5 e G7 obtiveram as menores somas totais, representando maior preferência de acordo com a Tabela de Newell e MacFarlane (FERREIRA et al., 2000), mas não diferiram significativamente entre si (Tabela 2). Contudo, a G3 foi estatisticamente semelhante a $G 4$ que não diferiu de forma significativa da G6 (a menos preferida). Pode-se, portanto, inferir que as geleias com maior proporção de manga foram as mais aceitas. MÉLO, LIMA E NASCIMENTO (1999) utilizando acerola na elaboração de geleias mistas com pitanga, observaram que as formulações contendo menor proporção de acerola apresentaram as melhores características sensoriais. Sendo assim, decidiu-se determinar o perfil das características sensoriais e físico-químicas dessas três geleias.

\section{TABELA 2 - RESULTADO DO TESTE DE ORDENAÇÃO QUANTO À PREFERÊNCIA DE GELEIA MISTA DE MANGA COM ACEROLA OBTIDA A PARTIR DE DIFERENTES FORMULAÇÕES}

\begin{tabular}{cc}
\hline GELEIAS MISTAS & Total \\
\hline G3 & $82^{\mathrm{bc}}$ \\
G4 & $115^{\mathrm{ab}}$ \\
G5 & $59^{\mathrm{c}}$ \\
G6 & $142^{\mathrm{a}}$ \\
\hline
\end{tabular}

Totais seguidos pela mesma letra não diferem significativamente entre si ao nível de $5 \%$ de probabilidade pelo teste de Friedman. Número de julgadores $=30$.

O perfil de características das geleias que atingiram o índice de preferência mais elevado (G3, G5 e G7) encontra-se na Figura 1. O atributo sabor acerola da G3 alcançou maior média que o da G5 e G7 e 
a análise estatística dos dados revelou diferença significativa entre as três formulações. O sabor manga mostrou-se mais perceptível na G5 e G7, as quais não diferiram estatisticamente entre si.

A G3 apresentou sabor mais ácido, consistência mais firme e menor brilho, diferindo estatisticamente da G5 e G7, as quais foram semelhantes entre si. Esse resultado corrobora o do teste de preferência e permite inferir que as geleias com maior percentual de manga em sua formulação reúnem as melhores características sensoriais.

\section{FIGURA 1- PERFIL SENSORIAL DAS GELEIAS MISTAS DE MANGA E ACEROLA}

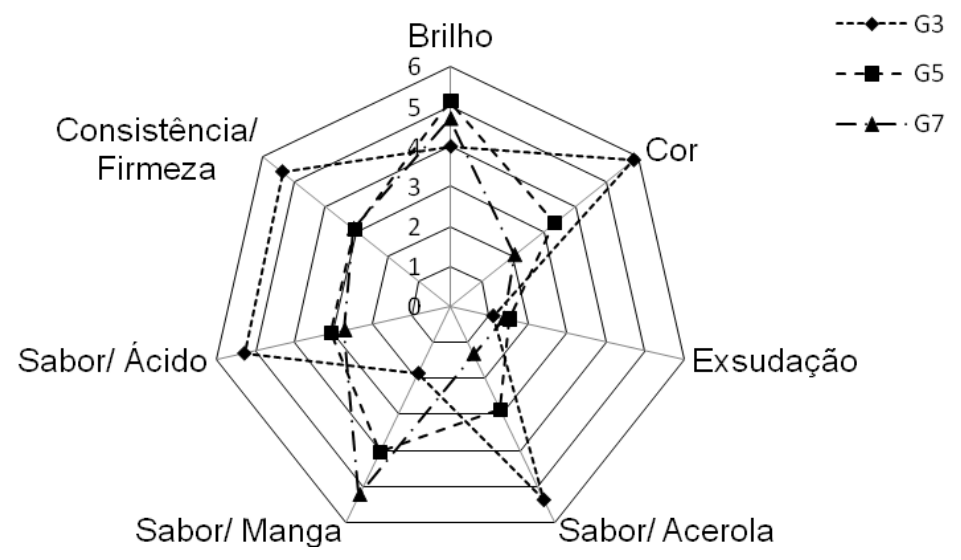

Atributos: brilho, cor, exsudação, sabor/acerola, sabor/manga, sabor/ácido e consistência/firmeza. G3: 50\% manga e 50\% acerola; G5: 60\% manga e 40\% acerola; G7: 75\% manga e 25\% acerola.

As características físico-químicas das polpas de acerola e de manga, antes do processamento, bem como das geleias simples e mista de manga e acerola encontram-se na Tabela 3. Frente ao teor de SST (63,5 a 64 Brix), todas as geleias podem ser classificadas como "extra" de acordo com a Resolução CNNPA n ${ }^{\circ} 12 / 78$ (BRASIL, 1978). $O$ pH das geleias simples e mista manteve-se entre 4,0 e 3,4, valores semelhantes aos encontrados por KANSCI, KOUBALA e LAPE (2003) em geleia de manga e por MACIEL et al. (1999) em geleia de acerola. Porém, é mais elevado do que o determinado por MÉLO, LINA E NASCIMENTO, (1999) em geleias mistas de acerola e pitanga. O pH da geleia de manga foi estatisticamente semelhante ao da polpa antes do processamento e significativamente mais elevado do que o da polpa de acerola, da geleia de acerola (100\%) e das geleias mistas (G3, G5 e G7).

A acidez total titulável (ATT) das geleias simples e mistas variou entre $0,7 \%$ e 2,9\%, porém enquadrou-se na faixa de acidez determinada para diferentes geleias de frutas (MÉLO, LIMA e NASCIMENTO, 1999; MACIEL et al., 1999; MOTA, 2006). Essas variações podem ser atribuídas às diferenças de acidez das frutas utilizadas e suas proporções nas formulações. A geleia de acerola (100\%), cuja fruta apresenta acidez mais elevada do que a manga revelou maior valor de ATT.

A geleia mista G3 (50\% de acerola) não apresentou diferença significativa $(p<0,05)$ para o teor de AA quando comparada com a geleia de acerola (100\%). Entretanto, mostrou-se significativamente mais elevado do que nas duas outras formulações (G5 e G7) e estatisticamente inferior ao da polpa da fruta. Ao comparar o teor de AA inicial (na polpa das frutas antes do processamento) verifica-se que nas geleias de acerola (100\%) e de manga (100\%) houve retenção de $67 \%$ e $69 \%$, respectivamente. Esses percentuais foram inferiores ao relatado por SILVA, LOPOS e VALENTE-MESQUITA (2006) ao avaliarem a estabilidade do ácido ascórbico em geleia de laranja (aproximadamente, 75\%). Constatase, portanto, que embora tenha ocorrido redução no teor de AA em função do processamento, as geleias mistas ainda apresentam elevado teor desse constituinte. Considerando que a recomendação 
diária de vitamina C para adultos e crianças é de respectivamente $100 \mathrm{mg} / \mathrm{dia}$ e $50 \mathrm{mg} / \mathrm{dia}$ (FNBNAS, 2000), a geleia mista de manga e acerola pode ser considerada boa fonte dessa vitamina.

\section{TABELA 3 - CARACTERÍSTICAS FÍSICO-QUÍMICAS DAS POLPAS, DAS GELEIAS SIMPLES E MISTAS DE MANGA E ACEROLA}

\begin{tabular}{|c|c|c|c|c|c|c|c|}
\hline DETERMNAÇÕES & PA & PM & GA & GM & G3 & G5 & G7 \\
\hline $\mathrm{pH}$ & $3,0 \pm 0,1$ & $3,8^{a} \pm 0,1$ & $3,4^{b} \pm 0,6$ & $4,0^{\mathrm{a}} \pm 0,1$ & $3,4^{b} \pm 0,0$ & $3,5^{b} \pm 0,0$ & $3,4^{b} \pm 0,0$ \\
\hline $\begin{array}{c}\text { SST } \\
\left({ }^{\circ} \text { Brix }\right)\end{array}$ & $6,8^{\mathrm{b}} \pm 0,7$ & $17,1^{\mathrm{c}} \pm 0,9$ & $64,0^{\mathrm{a}} \pm 0,0$ & $63,5^{a} \pm 0,0$ & $63,5^{a} \pm 0,5$ & $64,0^{\mathrm{a}} \pm 0,0$ & $64,0^{\mathrm{a}} \pm 0,0$ \\
\hline $\begin{array}{l}\text { ATT } \\
\text { (g de ácido málico } \\
\text { ou ctrico/ 100g) }\end{array}$ & $1,4^{a} \pm 0,1$ & $0,8^{\mathrm{c}} \pm 0,2$ & $2,9^{\mathrm{a}} \pm 0,1$ & $0,7^{\circ} \pm 0,3$ & $1,3^{\mathrm{b}} \pm 0,1$ & $0,9^{c} \pm 0,0$ & $1,2^{\mathrm{b}} \pm 0,0$ \\
\hline $\begin{array}{c}\mathrm{AA} \\
(\mathrm{mg} / 100 \mathrm{~g})\end{array}$ & $1.969^{\mathrm{a}} \pm 248,1$ & $262^{\mathrm{d}} \pm 35,2$ & $1.317^{\mathrm{b}} \pm 186,8$ & $180^{\mathrm{d}} \pm 29,5$ & $1.387^{\mathrm{b}} \pm 91,6$ & $768^{c} \pm 91,2$ & $659^{c} \pm 158,2$ \\
\hline $\begin{array}{c}\text { CAROTENOIDES } \\
\text { TOTAIS } \\
\text { ( } \mu \text { de equivalente } \\
\text { em } \beta \text {-caroteno/g) }\end{array}$ & $8,2^{c} \pm 0,9$ & $16,8^{\mathrm{a}} \pm 0,5$ & $1,21^{d} \pm 0,0$ & $12,7^{\mathrm{b}} \pm 2,8$ & $5,35^{\mathrm{c}} \pm 0,1$ & $5,65^{\mathrm{c}} \pm 0,2$ & $4,45^{\mathrm{c}} \pm 0,4$ \\
\hline $\begin{array}{l}\text { ANTOCANINAS } \\
(\mathrm{mg} / 100 \mathrm{~g})\end{array}$ & $4,6^{a} \pm 1,0$ & - & $1,9^{b} \pm 0,1$ & - & $2,3^{\mathrm{b}} \pm 0,3$ & $1,9^{\emptyset} \pm 0,8$ & $1,4^{b} \pm 0,4$ \\
\hline $\begin{array}{c}\text { FLAVONÓIS } \\
\text { TOTAIS } \\
\text { (mg de } \\
\text { quercetina/100 g) }\end{array}$ & $6,6^{c} \pm 0,6$ & $3,6^{\mathrm{d}} \pm 0,0$ & $10,4^{a} \pm 0,9$ & $5,4^{d} \pm 0,2$ & $9,3^{b} \pm 0,5$ & $9,6^{b} \pm 1,8$ & $7,9^{\mathrm{bc}} \pm 1,3$ \\
\hline $\begin{array}{c}\text { FENÓLICOS } \\
\text { TOTAIS } \\
\text { (mg de catequina/ } \\
100 \mathrm{~g} \text { ) }\end{array}$ & $1.450^{\mathrm{d}} \pm 240,13$ & $53,64^{c} \pm 22,7$ & $2.382^{a} \pm 140,6$ & $86,48^{c} \pm 15,3$ & $1.375^{\mathrm{b}} \pm 59,8$ & $1.046^{b} \pm 126,7$ & $1.111^{\mathrm{b}} \pm 139,4$ \\
\hline $\begin{array}{c}\text { LUMINOSID ADE - } \\
\text { L }\end{array}$ & $32,0^{a b} \pm 0,6$ & $50,8^{a} \pm 14,0$ & $29,4^{b} \pm 2,2$ & $39,1^{\mathrm{ab}} \pm 2,0$ & $30,8^{\mathrm{b}} \pm 10,2$ & $29,3^{b} \pm 3,0$ & $31,0^{\mathrm{b}} \pm 2,5$ \\
\hline COR $-\mathrm{a}$ & $12,6^{\mathrm{a}} \pm 0,5$ & $-0,24^{\mathrm{c}} \pm 4,6$ & $17,6^{a} \pm 2,8$ & $6,3^{b} \pm 1,3$ & $8,6^{\mathrm{b}} \pm 0,6$ & $6,9^{b} \pm 0,5$ & $5,2^{\mathrm{b}} \pm 0,7$ \\
\hline $\mathrm{COR}-\mathrm{b}$ & $14,0^{\mathrm{b}} \pm 0,5$ & $27,2^{\natural} \pm 3,7$ & $13,6^{\mathrm{b}} \pm 1,7$ & $20,9^{a} \pm 2,5$ & $16,2^{\mathrm{b}} \pm 5,2$ & $12,9^{b} \pm 2,1$ & $14,5^{\mathrm{b}} \pm 3,6$ \\
\hline
\end{tabular}

Médias seguidas pela mesma letra na linha não diferem entre si pelo teste de Tukey $(p<0,05)$. SST = sólidos solúveis totais; $\mathrm{ATT}=$ acidez total titulável; $\mathrm{AA}=$ ácido ascórbico; $\mathrm{PA}=$ polpa de acerola; $\mathrm{PM}=$ polpa de manga; $\mathrm{GA}=$ geleia de acerola $(100 \%) ; \mathrm{GM}=$ geleia de manga $(100 \%) ; \mathrm{G} 3=$ geleia mista $(50 \%$ manga e $50 \%$ acerola); G5 = geleia mista $(60 \%$ manga e $40 \%$ acerola); $\mathrm{G} 7$ = geleia mista ( $75 \%$ manga e $25 \%$ acerola).

A polpa de manga apresentou teor de carotenoides duas vezes maior do que a polpa de acerola, consequentemente as geleias de manga (100\%) e as mistas exibiram maior teor desse fitoquímico do que a de acerola (100\%). Ao comparar o teor de carotenoides totais da polpa de manga com a da geleia contendo $100 \%$ da fruta, evidencia-se retenção do pigmento da ordem de $75,7 \%$. Apesar do aumento na concentração de polpa de manga nas geleias G5 e G7, houve diminuição no teor de carotenoides em todas as geleias devido ao tratamento térmico, resultando em valores bem próximos que não apresentaram diferença significativa. GODOY e RODRIGUEZ-AMAYA (1987) e MERCADANTE e RODRIGUEZ-AMAYA (1998) observaram mudanças e/ou transformações entre derivados de carotenoides, ou em diferentes carotenoides após processamento e tratamento térmico de mangas em fatias ou purê. A presença de ácidos orgânicos, principalmente ácido ascórbico na polpa de acerola, pode ter contribuído para a proteção dos carotenoides na G3. Portanto, as geleias mistas além de fornecerem ácido ascórbico em elevadas quantidades veiculam considerável teor de carotenoides totais, fitoquímicos com reconhecida ação antioxidante. 
A cor vermelha da acerola decorre da presença de antocianinas, portanto esse pigmento foi detectado apenas nas geleias que continham essa fruta em sua composição e aquelas elaboradas com maiores proporções de acerola, consequentemente apresentaram maior teor desse fitoquímico. Ao comparar o teor de antocianinas da polpa de acerola com o das geleias, verificou-se redução desse pigmento, cujo percentual de retenção foi de 41,53\%; 51,42\%; 40,87\% e 31,20\% para a geleia de acerola (100\%) e as geleias mistas G3, G5 e G7, respectivamente. Vários fatores podem ter contribuído para a degradação desse pigmento, a exemplo da presença de oxigênio, de ácido ascórbico, de hidroximetilfurfural, bem como o aquecimento utilizado no processo (BOBBIO e BOBBIO, 2003). Outros autores (GARCÍA-VIGUERA et al., 1998; MOTA, 2006; PLESSI, BERTERLLI e ALBASINI, 2007) relatam, também, redução nos teores de antocianinas em geleias de frutas.

A polpa de manga apresenta valores de flavonóis e fenólicos totais bem abaixo da polpa de acerola. Ao comparar o teor desses compostos nas polpas com as geleias $(100 \%)$ das respectivas frutas, evidencia-se aumento significativo desse constituinte na geleia de acerola, diferentemente do que ocorreu com geleia de manga. Resultados semelhantes foram encontrados por PLESSI, BERTERLLI e ALBASINI (2007) e ZAFRILLA, FERRERAS e TOMÁS-BARBERÁN (2001) em geleias de frutas do gênero Rubus e Ribes e por $\mathrm{OBOH}$ (2005) em folhosos. Esse aumento se deve ao aquecimento que, provavelmente, facilita a extração do fitoquímico, além de promover a quebra de taninos presentes nos vegetais em fenóis simples. Por outro lado, HÄKKINEN et al. (2000) observaram que os flavonóis presentes no morango sofreram pequenas alterações após o processamento de geleias e ressaltaram que provavelmente a adição de açúcar tenha protegido esses compostos durante a cocção. Resultados semelhantes foram encontrados por GARCIA-VIGUERA et al. (1993) no processamento de geleias de citros. Frente à diversidade química desses fitoquímicos, acredita-se que os mesmos apresentam sensibilidade diferenciada quando expostos às condições de processamento.

Segundo PRATI, MORETTI e CARDELLO (2005), na determinação objetiva da cor dos produtos, o valor $L$ expressa a luminosidade ou claridade da amostra e pode variar de 0 a 100 . Assim sendo, quanto mais próximo ou mais distante de 100 , mais clara ou mais escura será a amostra. Por sua vez, valores positivos de a mais elevados indicam tendência à coloração vermelha e os negativos coloração verde, enquanto que os valores positivos de b expressam maior intensidade de amarelo e os negativos maior intensidade de azul.

As geleias simples e mistas não diferiram estatisticamente quanto aos valores de $L$, a e b, mas o de $L$ (em torno de 30 ) demonstra que a cor apresenta tonalidade escura. O valor de a, entre 5,17 a 8,63, indica a presença reduzida da coloração vermelha e o de b, entre 12,87 e 16,21, revela a leve presença da cor amarela. A cor da polpa de manga sobressaiu-se em todas as geleias mistas, independente da proporção em que estava presente. Essa característica torna-se interessante uma vez que os resultados da análise sensorial mostraram a preferência pelas geleias com maiores concentrações de manga.

\section{CONCLUSÃO}

As geleias G7 (75\% manga e $25 \%$ acerola) e a G5 (60\% manga e $40 \%$ acerola) foram as mais apreciadas pelos julgadores, demonstrando que a manga em maior proporção na formulação confere melhores características sensoriais. Essas geleias em função dos teores de ácido ascórbico, carotenoides totais e de polifenóis (fenólicos, antocianinas e flavonóis totais) podem ser consideradas promissoras por apresentarem forte potencial antioxidante. Assim, a polpa de acerola com teores significativos desses fitoquímicos bioativos pode ser vista como importante agente enriquecedor na produção de geleias mistas com manga.

\section{ABSTRACT}

SENSORY AND PHYSICO-CHEMICAL CHARACTERIZATIONS OF MIXED MANGO AND ACEROLA JAM

This work has as objective to determine the best formulation of mixed mango and acerola jam and to verify the effects of processing on the physico-chemical and sensory properties of the product. Five formulations containing different concentrations of mango juice were formulated $(\mathrm{M})(75 \%, 60 \%, 50 \%, 40 \%, 25 \%)$, acerola (A) $(25 \%, 40 \%$, 
$50 \%, 60 \%, 75 \%)$, pectin $(0.1 \% ; 0.125 \% ; 0.2 \% ; 0.3 \% ; 0.6 \%)$, citric acid $(0.1 \% ; 0.125 \% ; 0.25 \%)$ and sugar $(30 \%)$. The obtained jams were submitted to a sensory test by the method of preference by ranking and those more acceptable were analyzed by Descriptive Quality Analysis. Total titratable acidity (TTA), pH, Ascorbic acid (AA), Total soluble solids (TSS), Anthocyanins, Flavonoids, Total Carotenoids Phenolics and Color were carried out and the results were analyzed by ANOVA and Tukey test at $5 \%$. All the jams were classified as "extra", with TSS between 63.5 to $64{ }^{\circ}$ Brix. The jam, J7 (75\%M:25\%A) was the most acceptable by the judges followed by J5 (60\%M:40\%A) and J3 (50\%M:50\%A). Mixed jams showed high levels of ascorbic acid and significant values of carotenoids and poliphenols. Jams with greater concentration of mango were more accepted by the judges and showed a promissory antioxidant function based on the levels of ascorbic acid, total carotenoids and polyphenols. Therefore, acerola, (with significant levels of bioactive phytochemical compounds) can be seen as an enriched agent in the production of mango mixed fruit jam.

KEY-WORDS: MIXED JAM; MANGO; ACEROLA; SENSORY ANALYSIS; ASCORBIC ACID; PHYTOCHEMICALS.

\section{REFERÊNCIAS}

1 AGOSTINI-COSTA, T. S.; ABREU, L. N.; ROSSETTI, A. G. Efeito do congelamento e do tempo de estocagem da polpa de acerola sobre o teor de carotenóides. Revista Brasileira Fruticultura, Jaboticabal, v. 25, n. 1, p. 56-58, 2003.

2 ARANHA, F. Q.; MOURA, L. S. A; SIMÕES, M. O. S; BARROS, Z. F.; QUIRINO, I. V. L.; METRI, J. C; BARROS, J. C. Normalização dos níveis séricos de ácido ascórbico por suplementação com suco de acerola (Malpighia glabra L.) ou farmacológica em idosos institucionalizados. Revista Nutrição, Campinas, v. 17, n. 3, p. 309-317, 2004.

3 ASSIS, S.A.; MARTINS, A.B.G.; GUAGLIANONI, D.G.; OLIVEIRA, O.M.M.F. Partial purification and characterization of pectin methylesterase from acerola (Malpighia glabra L.). Journal of Agricultural Food Chemistry, Easton, v. 50, p. 4103-4107, 2002.

4 AOAC. Association of Official Analytical Chemists. Official methods of analysis of AOAC International. $17^{\text {th }}$ ed. Arlington, 2002. v. 2.

5 BARNABÉ, D; VENTURINI FILHO, W.G. Características físico-químicas e sensoriais de refrigerantes de acerola produzidos a partir de suco desidratado e extrato seco da fruta. Journal of Food Technology, Oxford, v. 7, p.6976, 2004

6 BASF S.A. Unidades de Produtos para Fruticultura. Frutas para exportação. Atualidades agrícolas: fruticultura o sucesso do Vale São Francisco, São Bernardo do Campo, n. 6, p.16-29, jun.2006.

7 BOBBIO, F. O.; BOBBIO, P. A. Química de alimentos. São Paulo: Varela. 2003. 240 p.

8 BRASIL. Ministério da Saúde. Resolução CNNPA n. 12 de 24 de julho de 1978. Normas técnicas e especiais para alimentos e bebidas. Disponível em:<http://www.anvisa.gov.br/legisl/resol/12_78_guarana.htm> Acesso em: 20 mar. 2008

9 CARDELLO, H. M. A. B.; CARDELLO, L. Teor de vitamina c, atividade de ascorbato oxidase e perfil sensorial de manga (mangífera índica l.) var. haden, durante o amadurecimento. Ciência e Tecnologia de Alimentos, Campinas, v. 18, n. 2, p. 211-217, 1998.

10 DUTRA E. S. Fabricação de geléia: goiaba e morango. Brasília: SENAR, 2000. 91 p.

11 FERREIRA, V. L. P.; ALMEIDA, T. C. A.; PETTINELLI, M. C. V.; SILVA, M. A. P.; CHAVES, J. B. P.; BARBOSA, E. M. M. Análise sensorial: testes discriminativos e afetivos. Campinas, SP: SBCTA, 2000. 127 p.

12 FNBNA. Food and Nutrition Board of the National Academy of Science. Dietary reference intakes for Vitamin C, Vitamin E, Selenum and carotenoids. Washington: National Academy Press, 2000, 15 p.

13 GARCÍA-VIGUERA, C.; ZAFRILLA, P.; ARTÉS, F.; ROMERO, F.; ABELLÁM, P.; TOMÁS-BARBERAN, F.A. Colour and anthocyanin stability of red raspberry jam. Journal Science of Food and Agricultural, Easton, v. 78, p. 556-573, 1998.

14 GARCIA-VIGUERA, C.; BARBERÁN, F.A.T.; FERRERES, F.; ARTÉS, F.A. Determination of citrus jams genuiness by flavonoid analysis. Zeitschrift für lebens mittel-Untersuchung und-Forchung, Berlim, v.197, p. 255-259, 1993.

15 GODOY, H. T.; RODRIGUEZ-AMAYA, D. B. Changes in individual carotenoids on processing and storage of mango (Mangifera indica) slices and purée. International Journal Food Science and Technology, Oxford, v. 22. p. 451460, 1987.

16 HÄKKINEN, S.H.; KÄRENLAMPI, S.O.; MYKKÄNEN, H.M.; TÖRRÖNEN, A.R. Influence of domestic processing and storage on flavonol contents in berries. Journal of Agricultural and Food Chemistry, London, v. 48, n. 7, p. 2960-2965, 2000.

17 JACKIX, M.H. Doces, geléias e frutas em calda. Campinas-SP: UNICAMP/Ícone. 1988. 172 p. 
18 JACOMETTI, G. A.; MENEGHEL, R. F. A.; YAMASHITA, F. Aplicação de revestimentos comestíveis em pêssego (Prunus persica). Ciência e Tecnologia de Alimentos, Campinas, v. 23, n. 1, p. 95-100, 2003.

$19 \mathrm{KANSCI}, \mathrm{G}$; KOUBALA, B. B.; LAPE, I. M. Effect of ripening on the composition and the suitability for jam processing of different varieties of mango (Mangifera indica). African Journal of Biotechnology, Abraka, v. 2, n. 9, p. 301-306, 2003.

20 KUSKOSKI, M. E.; ASUERO, A. G.; TRONCOSO, A. M.; MANCINI-FILHO, J.; FETT, R. Aplicación de diversos métodos químicos para determinar actividad antioxidante en pulpa de frutos. Ciência e Tecnologia de Alimentos, Campinas, v. 25, n. 4 , p. $726-732,2005$.

21 LEES, D. H.; FRANCIS, F. J. Standardization of pigment analyses in cranberries. HortScience, Alexandria, v. 7, n. 1, p. 83-84, 1972

22 LIMA, V. L. A. G.; MÉLO, E. A.; MACIEL, M. I. S.; LIMA, D. E. S. Avaliação do teor de antocianinas em polpa de acerola congelada proveniente de frutos de 12 diferentes aceroleiras (Malpighia emarginata D. C.). Ciência e Tecnologia de Alimentos, Campinas, v. 23, n. 1, p. 101-103. 2003.

23 MACIEL, M. I. S.; MELO E.A.; LIMA, V. L. A. G. de; SILVA, M. R. F da. Processing and storage of acerola (Malpighia sp) fruit and its products. Food Science Technology, v. 36, n. 2, p. 142-146, 1999.

24 MATSUURA, F. C. A. U.; FOLEGATTI, M. I. S.; CARDOSO, R. L.; FERREIRA, D. C. Sensory acceptance of mixed nectar of papaya, passion fruit and acerola. Scientia Agrícola, Piracicaba, v. 61, n. 6, p. 604-608, 2004.

25 MATSUURA, F.C.A.U.; ROLIM, R.B. Avaliação da adição de acerola em suco de abacaxi visando à produção de um "blend" com alto teor de vitamina C. Revista Brasileira de Fruticultura, Jaboticabal, v. 24, n. 1, p. 138-141, 2002.

26 McGUIRE, R. G. Reporting of objective color measurements. HortScience, Alexandria, v. 27, n.12, p. 1254 -1255, 1992.

27 MELO, E. A; LIMA. V.L.A.G.; NASCIMENTO, P.P. Formulação e avaliação físico-química e sensorial de geléia mista de pitanga (Eugenia uniflora L.) e acerola ( Malpighia sp). Boletim do CEPPA, Curitiba, v. 17, n.1, p. 33-44, 1999.

28 MERCADANTE, A. Z.; RODRIGUEZ-AMAYA, D. B. Effects of ripening, cultivar differences, and processing on the carotenoid composition of mango. Journal of Agricultural and Food Chemistry, Washington, v. 46. p. 128-130, 1998.

29 MOTA, R. V. Caracterização física e química de geléia de amora-preta. Revista Ciência e Tecnologia de Alimentos, Campinas, v.26, n. 3, p. 539-543, 2006.

$30 \mathrm{OBOH}, \mathrm{G}$. Effect of blanching on the antioxidant properties of some tropical green leafy vegetables. LWT - Food Science and Technology, Zurich, v.38, n.5, p. 513-517, 2005.

31 PENNA, E.W. Evaliación sensorial: una metodología para tecnología de alimentos. Santiago: Universidade do Chile, 1980. $134 \mathrm{p}$.

32 PLESSI, M.; BERTERLLI, D.A.; ALBASINI, A. Distribution of metalsand phenolic compounds as a criterion to evaluate variety of berries and related jams. Food Chemistry, London, v.100, p. 419-427, 2007.

33 PRATI, P.; MORETTI, R.H.; CARDELLO, H.M.A.M. Elaboração de bebida composta por mistura de garapa parcialmente clarificada-estabilizada e suco de frutas ácidas. Revista Ciência e Tecnologia de Alimentos, Campinas, v. 25, n. 1, p. 147-152, 2005.

34 RODRIGUEZ-AMAYA, D. B. A guide to carotenoid analysis in foods. Washington: ILSI Press, 1999.64 p.

35 SILVA, P. T.; LOPES, M.L.M.; VALENTE-MESQUITA, V. L. Efeito de diferentes processamentos sobre o teor de ácido ascórbico em suco de laranja utilizado na elaboração de bolo, pudim e geléia. Revista Ciência e Tecnologia de Alimentos, Campinas, v.26, n.3, p. 678-682, 2006.

36 STONE, H.; SIDEL, J. Sensory evaluation practices. New York: Academic Press, 1993.338 p.

37 TSIMIDOU, M.; PAPADOPOULOS, G.; BOSKOU, D. Phenolic compounds and stability of virgin olive oil - part I. Food Chemistry, London, v. 45, p.141-144, 1992.

38 ZAFRILLA, P.; FERRERAS, F.; TOMÁS-BARBERÁN, F.A. Effect of processing and storage on the antioxidant ellagic acid derivatives and flavonoids of red raspberry (Rubus idaeus) jams. Journal of Agricultural and Food Chemistry, Easton, v. 49, p. 3651-3655, 2001.

\section{AGRADECIMENTOS}

À Universidade Federal Rural de Pernambuco (UFRPE) e ao CNPq pelo apoio financeiro através do Edital MCT/MESA/CNPq/CT - Agronegócio e FACEPE/PROMATA. 